\title{
Equations of low-degree Projective Surfaces with three-divisible Sets of Cusps
}

\author{
Wolf P. Barth Sławomir Rams
}

\begin{abstract}
We determine the equations of surfaces $Y \subset \mathbf{P}_{3}(\mathbf{C})$ of degrees $\leq 6$ carrying a minimal, non-empty, three-divisible set of cusps.
\end{abstract}

\section{Introduction}

We consider algebraic surfaces $Y \subset \mathbb{P}_{3}(\mathbb{C})$. A cusp (=singularity $\left.A_{2}\right)$ on $Y$ is a singularity near which the surface is given in local (analytic) coordinates $x, y$ and $z$, centered at the singularity, by an equation

$$
x y-z^{3}=0 .
$$

This is an isolated quotient singularity $\mathbb{C}^{2} / \mathbb{Z}_{3}$. A set $P_{1}, \ldots, P_{n}$ of cusps on $Y$ is called 3-divisible, if there is a cyclic global triple cover of $Y$ branched precisely over these cusps. Equivalently: If $\pi: X \rightarrow Y$ is the minimal desingularization introducing two $(-2)$-curves $E_{\nu}^{\prime}, E_{\nu}^{\prime \prime}$ over each cusp, then there is a way to label these curves such that the divisor class of

$$
\sum_{\nu=1}^{n}\left(2 E_{\nu}^{\prime}+E_{\nu}^{\prime \prime}\right)
$$

is divisible by 3 in $N S(X)$ [T, B 1]. The aim of this note is to determine the equations for surfaces $Y \subset \mathbb{P}_{3}$ of degrees $\leq 6$ carrying a minimal, non-empty set of 3-divisible cusps. Applications of 3 -divisible sets can be found in $[\mathrm{T}]$, [KZ].

Recall $[\mathrm{T}]$ that a non-empty 3 -divisible set of cusps on a surface of degree $d$ contains $n$ points with

\begin{tabular}{l|llc}
$d$ & 3 & 4 & 5 \\
\hline$n$ & 3 & 6 & $\geq 12$
\end{tabular}

Here we show

Theorem 1.1 Any non-empty 3-divisible set of cusps on a sextic surface contains at least 18 points.

Suported by the DFG Schwerpunktprogramm "Global methods in complex geometry". The second author is supported by a Fellowship of the Foundation for Polish Science and KBN Grant No. 2 P03A 01625.

2000 Mathematics Subject Classification. 14J25, 14J17. 
The cubic surface $Y \subset \mathbb{P}_{3}$ with three cusps is unique, up to a choice of coordinates it has the equation $x_{1} x_{2} x_{3}-x_{0}^{3}=0[\mathrm{~T}]$. For surfaces $Y \subset \mathbb{P}_{3}$ of degrees $\mathrm{d}=4,5,6$ with a 3-divisible set of $6,12,18$ cusps we show (see lemma 2.1 and theorem 2.2):

Theorem. There are two polynomials $s^{\prime}, s^{\prime \prime}$ of degree 3 and some polynomial s of degree 2 such that the sextic polynomial $s^{\prime} \cdot s^{\prime \prime}-s^{3}$ vanishes on $Y$. The equation $s^{\prime} \cdot s^{\prime \prime}-s^{3}=0$ in the case of degree

$d=4$ : can be chosen such that it defines the quartic $Y$ together with some residual quadric;

$d=5$ : defines the quintic $Y$ together with a residual plane;

$d=6$ : either defines the sextic $Y$ or it vanishes identically. If the latter holds then $Y$ has an equation $l^{\prime} \cdot l^{\prime \prime} \cdot f-g^{3}=0$ with $\operatorname{deg}\left(l^{\prime}\right)=\operatorname{deg}\left(l^{\prime \prime}\right)=1, \operatorname{deg}(g)=2, \operatorname{deg}(f)=4$.

This description allows to conclude:

- Quartic surfaces with six irreducible cusps form an irreducible family. Based on the equation given above, this has been shown in $[R]$.

- Quintic surfaces with 12 three-divisible cusps form an irreducible family (thm.2.1).

- Sextic surfaces with 18 three-divisible cusps form two irreducible families. In fact the equations for the surfaces in the two families are of the two types given in the theorem above. That these two families are disjoint, this follows quite easily when considering the codes of these surfacesi $[\mathrm{BR}]$.

Convention: In this note the base field always is $\mathbb{C}$. When we consider singular surfaces, by a divisor we mean a Weil-divisor.

\section{Contact cubics}

Let $Y \subset \mathbb{P}_{3}$ be a surface of degree $d=4,5$ or 6 . Let $P_{1}, \ldots, P_{n} \in Y$ be a 3 -divisible set of $n=6,12$ or $\leq 18$ cusps. We assume that $Y$ is smooth but for these cusps, and perhaps for some further rational double points. The aim of this section is to show that there are two cubic surfaces touching $Y$ to the third order along two curves passing through the n cusps.

We denote by

$$
\pi: X \rightarrow Y
$$

the minimal resolution of singularities. In this situation it is well-known, that the canonical bundle of $X$ is

$$
K_{X}=\pi^{*}\left(\mathcal{O}_{Y}(d-4)\right) .
$$

By abuse of notation we write $\mathcal{O}_{X}(k):=\pi^{*} \mathcal{O}_{Y}(k), k \in \mathbb{Z}$. 
The minimal desingularisation introduces two (-2)-curves $E_{\nu}^{\prime}$ and $E_{\nu}^{\prime \prime}$ over each cusp $P_{\nu}$. We label them such that the two classes

$$
\mathcal{L}^{\prime}:=\frac{1}{3} \sum_{\nu=1}^{n}\left(2 E_{\nu}^{\prime}+E_{\nu}^{\prime \prime}\right) \quad \text { and } \quad \mathcal{L}^{\prime \prime}:=\frac{1}{3} \sum_{\nu=1}^{n}\left(E_{\nu}^{\prime}+2 E_{\nu}^{\prime \prime}\right)
$$

exist in $N S(X)$. We also introduce the divisor classes

$$
\mathcal{C}^{\prime}:=\mathcal{O}_{X}(1)-\mathcal{L}^{\prime}, \mathcal{C}^{\prime \prime}:=\mathcal{O}_{X}(1)-\mathcal{L}^{\prime \prime}, \quad \mathcal{D}^{\prime}:=\mathcal{O}_{X}(1)+\mathcal{L}^{\prime}, \mathcal{D}^{\prime \prime}:=\mathcal{O}_{X}(1)+\mathcal{L}^{\prime \prime}
$$

on $X$. Obviously

$$
\left(\mathcal{L}^{\prime} \cdot E_{\nu}^{\prime}\right)=-1, \quad\left(\mathcal{L}^{\prime} \cdot E_{\nu}^{\prime \prime}\right)=0
$$

and similarly for $\mathcal{L}^{\prime \prime}$. This implies

$$
\left(\mathcal{L}^{\prime}\right)^{2}=\left(\mathcal{L}^{\prime \prime}\right)^{2}=-\frac{2}{3} n, \quad\left(\mathcal{C}^{\prime}\right)^{2}=\left(\mathcal{C}^{\prime \prime}\right)^{2}=\left(\mathcal{D}^{\prime}\right)^{2}=\left(\mathcal{D}^{\prime \prime}\right)^{2}=d-\frac{2}{3} n .
$$

The assertions stated for $\mathcal{L}^{\prime}$ in the next lemma similarly hold for $\mathcal{L}^{\prime \prime}$.

Lemma 1.1 a) The class $\mathcal{L}^{\prime}$ in $N S(X)$ is not an integral linear combination

$$
E+\sum_{\nu=1}^{n}\left(a_{\nu}^{\prime} E_{\nu}^{\prime}+a_{\nu}^{\prime \prime} E_{\nu}^{\prime \prime}\right), \quad a_{\nu}^{\prime}, a_{\nu}^{\prime \prime} \in \mathbb{Z}
$$

with $E$ an exceptional divisor lying over the additional singularities of $Y$.

b) The class $\mathcal{L}^{\prime}$ is not effective.

c) [R 2, lemma 2.1] If $C^{\prime} \in\left|\mathcal{C}^{\prime}\right|$, then $\pi\left(C^{\prime}\right) \subset Y$ cannot be a plane section.

Proof. a) If $\mathcal{L}^{\prime}$ is represented by an integral linear combination $L$ as in the assertion, we find

$$
-1=\left(L \cdot E_{\nu}^{\prime}\right)=a_{\nu}^{\prime \prime}-2 a_{\nu}^{\prime}, \quad 0=\left(L \cdot E_{\nu}^{\prime \prime}\right)=a_{\nu}^{\prime}-2 a_{\nu}^{\prime \prime} .
$$

This would imply $a_{\nu}^{\prime}=2 a_{\nu}^{\prime \prime}$ and lead to the contradiction $a_{\nu}^{\prime \prime}=\frac{1}{3}$.

b) From $\left(\mathcal{O}_{X}(1) \cdot E_{\nu}^{\prime}\right)=\left(\mathcal{O}_{X}(1) \cdot E_{\nu}^{\prime \prime}\right)=0$ we conclude $\left(\mathcal{O}_{X}(1) \cdot \mathcal{L}^{\prime}\right)=0$. If $L$ is an effective divisor representing $\mathcal{L}^{\prime}$, then $\left(\mathcal{O}_{X}(1) . L\right)=0$ implies that $L$ is an integral linear combination of exceptional divisors lying over the singularities of $Y$. This is in conflict with a).

c) Assume that $\pi\left(C^{\prime}\right)$ is a plane section. The total transform of this plane section on $X$ then is a divisor $C^{\prime}+E$ with $E$ an integral linear combination of exceptional divisors. Hence

$$
\mathcal{O}_{X}(1) \sim C^{\prime}+E \sim \mathcal{O}_{X}(1)-\mathcal{L}^{\prime}+E, \quad \text { so } \quad \mathcal{L}^{\prime} \sim E,
$$

a contradiction with a).

We use Riemann-Roch on $X$

$$
\chi\left(\mathcal{C}^{\prime}\right)=\chi\left(\mathcal{O}_{X}\right)+\frac{1}{2} \mathcal{C}^{\prime} \cdot\left(\mathcal{C}^{\prime}-K_{X}\right)
$$


and similarly for $\mathcal{C}^{\prime \prime}$. Here $\chi\left(\mathcal{O}_{X}\right)$ is the Euler-characteristic $\chi\left(\mathcal{O}_{Y^{\prime}}\right)$ for any smooth surface $Y^{\prime}$ of degree $d$, since $X$ and $Y^{\prime}$ are diffeomorphic $[\mathrm{Br}]$. We obtain the table

\begin{tabular}{c|cccc|c}
$d$ & $K_{X}$ & $\chi\left(\mathcal{O}_{X}\right)$ & $\left(\mathcal{C}^{\prime}\right)^{2}$ & $-\mathcal{C}^{\prime} . K_{X}$ & $\chi\left(\mathcal{C}^{\prime}\right)$ \\
\hline 4 & $\mathcal{O}_{X}$ & 2 & 0 & 0 & 2 \\
5 & $\mathcal{O}_{X}(1)$ & 5 & -3 & -5 & 1 \\
6 & $\mathcal{O}_{X}(2)$ & 11 & $6-\frac{2}{3} n$ & -12 & $8-\frac{n}{3}$
\end{tabular}

The main fact we need is this:

Proposition 1.1 The divisor classes $\mathcal{C}^{\prime}$ and $\mathcal{C}^{\prime \prime}$ on $X$ are effective.

Proof. It suffices to prove the assertion for $\mathcal{C}^{\prime}$. This follows from Riemann-Roch by controlling $h^{2}\left(\mathcal{C}^{\prime}\right)$. The proof is easy for $d=4$ or 5 , but quite tedious for $d=6$. We consider these three cases.

$d=4$ : Here $h^{2}\left(\mathcal{C}^{\prime}\right)=h^{0}\left(-\mathcal{C}^{\prime}\right)=h^{0}\left(\mathcal{O}_{X}(-1)+\mathcal{L}^{\prime}\right)$. Since $\mathcal{O}_{X}(1)$ is nef, from $\left(-\mathcal{C}^{\prime} . \mathcal{O}_{X}(1)\right)=$ -4 it follows that $-\mathcal{C}^{\prime}$ cannot be effective. So $h^{2}\left(\mathcal{C}^{\prime}\right)=0$ and $h^{0}\left(\mathcal{C}^{\prime}\right) \geq 2$.

$d=5$ : Now $h^{2}\left(\mathcal{C}^{\prime}\right)=h^{0}\left(\mathcal{O}_{X}(1)-\mathcal{C}^{\prime}\right)=h^{0}\left(\mathcal{L}^{\prime}\right)=0$ by lemma $\left.1.1 \mathrm{~b}\right)$.

So let us now concentrate on the case $d=6$. Since $K_{X}=\mathcal{O}_{X}(2)$, by Serre-duality

$$
h^{2}\left(\mathcal{C}^{\prime}\right)=h^{0}\left(\mathcal{D}^{\prime}\right), \quad h^{2}\left(\mathcal{C}^{\prime \prime}\right)=h^{0}\left(\mathcal{D}^{\prime \prime}\right)
$$

The effective class $E:=\sum\left(E_{\nu}^{\prime}+E_{\nu}^{\prime \prime}\right)$ defines a map $\mathcal{C}^{\prime} \rightarrow \mathcal{D}^{\prime \prime}$ and an exact sequenc

$$
0 \rightarrow \mathcal{C}^{\prime} \rightarrow \mathcal{D}^{\prime \prime} \rightarrow \mathcal{D}^{\prime \prime} \mid E \rightarrow 0
$$

From $\left(\mathcal{D}^{\prime \prime} . E_{\nu}^{\prime}\right)=0,\left(\mathcal{D}^{\prime \prime} . E_{\nu}^{\prime \prime}\right)=-1$ we conclude $h^{0}\left(\mathcal{D}^{\prime \prime} \mid E\right)=0$. This implies

$$
h^{0}\left(\mathcal{C}^{\prime}\right)=h^{0}\left(\mathcal{D}^{\prime \prime}\right)=h^{2}\left(\mathcal{C}^{\prime \prime}\right) .
$$

So proposition 1.1 follows from Riemann-Roch even in the case $d=6$ and $n \leq 18$ if we show

Proposition 1.2 If $d=6$, then $h^{0}\left(\mathcal{C}^{\prime}\right) \leq 1, h^{0}\left(\mathcal{C}^{\prime \prime}\right) \leq 1$.

Proof. As usual this statement needs to be proven for $\mathcal{C}^{\prime}$ only. So let us assume the assertion to be false, and $h^{0}\left(\mathcal{C}^{\prime}\right) \geq 2$. We consider the linear system $\left|\mathcal{C}^{\prime}\right|$ on $X$ with

$$
\mathcal{C}^{\prime} \cdot \mathcal{O}_{X}(1)=6, \quad\left(\mathcal{C}^{\prime}\right)^{2}=6+\left(\mathcal{L}^{\prime}\right)^{2} \geq-6 .
$$

Let $B$ be the base curve of the system and $|F|$ its free part. Then $F^{2} \geq 0$ and

$$
1 \leq\left(F . \mathcal{O}_{X}(1)\right) \leq\left(\mathcal{C} . \mathcal{O}_{X}(1)\right)=6 .
$$

Lemma 1.2 We have $F^{2}=0,2$ or 4 . If $F^{2}=2$, then $\left(F \cdot \mathcal{O}_{X}(1)\right) \geq 4$ and if $F^{2}=4$, then $\left(F . \mathcal{O}_{X}(1)\right) \geq 5$. 
Proof. Since $K_{X}=\mathcal{O}_{X}(2)$ is a square, from Riemann Roch it follows that all divisor classes on $X$ have an even self-intersection. We consider the determinant

$$
\operatorname{det}\left(\begin{array}{cc}
\mathcal{O}_{X}(1)^{2} & \left(F . \mathcal{O}_{X}(1)\right) \\
\left(F . \mathcal{O}_{X}(1)\right) & F^{2}
\end{array}\right)=6 \cdot F^{2}-\left(F \cdot \mathcal{O}_{X}(1)\right)^{2} .
$$

Since $\left(F \cdot \mathcal{O}_{X}(1)\right) \leq 6$, the Hodge index theorem implies $F^{2} \leq 6$. And if $F^{2}=6$, then $F \sim r \mathcal{O}_{X}(1)$ with some rational number $r$. From $6 r=\left(F \cdot \mathcal{O}_{X}(1)\right)=6$ we conclude $r=1$ and $F \sim \mathcal{O}_{X}(1)$. But this implies

$$
\mathcal{O}_{X}(1)-\mathcal{L}^{\prime}=\mathcal{O}_{X}(1)+B
$$

and $-\mathcal{L}^{\prime} \sim B$ would be effective. Since $3 \mathcal{L}^{\prime}$ is effective, this is a contradiction.

We are left with the cases $F^{2}=0,2$ or 4 . In these cases the class of $F$ cannot be a rational multiple of $\mathcal{O}_{X}(1)$. By the Hodge index theorem the above determinant must be negative. This implies $\left(F \cdot \mathcal{O}_{X}(1)\right) \geq 4$ for $F^{2}=2$ and $\geq 5$ for $F^{2}=4$.

Lemma 1.3 The general curve $F_{0} \in|F|$ is irreducible.

Proof. Assume that all curves $F_{0} \in|F|$ are reducible. First we show that then $F \sim k A, 2 \leq$ $k \in \mathbb{N}$, with some effective irreducible curve $A \subset X$. To do this, blow up the base points of $|F|$ via $\sigma: X^{\prime} \rightarrow X$. (If $F^{2}=0$ there are no base points.) Let $\left|F^{\prime}\right|$ on $X^{\prime}$ be the proper transform of the linear system $|F|$. If $\left(F^{\prime}\right)^{2}>0$, by Bertini the general curve $F_{0}^{\prime} \in\left|F^{\prime}\right|$ will be irreducible, and this implies that the general curve $F_{0}$ is irreducible. So we are in the case $\left(F^{\prime}\right)^{2}=0$. Then $F^{\prime}$ is composed of a pencil. All irreducible components $A_{i}$ of a general fibre $F_{0}^{\prime}$ are algebraically equivalent, and by $q\left(X^{\prime}\right)=0$ linearly equivalent. This implies $F_{0} \sim k A^{\prime}$ with $A^{\prime}$ such an irreducible component and $k$ the number of components in a fibre. But with $A:=\sigma\left(A^{\prime}\right)$ we get

$$
F_{0} \sim \sigma\left(F_{0}^{\prime}\right) \sim k A
$$

Now consider the different cases: If $F^{2}=2$, then $F$ cannot be linearly equivalent to some $k \cdot A$, because then $F^{2} \geq 4 A^{2}$. And if $F^{2}=4$, then $F^{2}=k^{2} A^{2}$ would imply $k=2$ and $A^{2}=1$. This is impossible, because all self-intersections of curves on $X$ are even numbers. We are left with the case $F^{2}=0$. Here $F \sim k \cdot A$ with a smooth irreducible curve $A \subset X$. The degree of its (birational) image $\pi(A) \subset \mathbb{P}_{3}$ can be at most $6 / 2=3$. So $A$ will be rational or elliptic. But this is in conflict with the adjunction formula

$$
2 g(A)-2=2\left(A \cdot \mathcal{O}_{X}(1)\right)
$$

and $\left(A . \mathcal{O}_{X}(1)\right) \geq 1$.

Lemma 1.4 The image curve $\pi(A)$ of a general $A \in|F|$ is a plane quintic.

Proof. Put $a:=\left(A \cdot \mathcal{O}_{X}(1)\right)$. Here $a>0$, since $A$ is not exceptional. It suffices to prove that $\pi(A)$ is planar irreducible. The adjunction formula for the plane curve $\pi(A)$ then shows

$$
A^{2}+2 a=2 p_{a}(A)-2 \leq a(a-3) .
$$


From $A^{2} \geq 0$ we conclude $a^{2}-5 a \geq 0$. So $a \geq 5$ and $a=6$ only if $A$ is a plane section of $Y$. But the latter would contradict lemma $1.1 \mathrm{c}$ ).

If $F^{2}=0$, the general fibre $A$ of $F$ is smooth by Bertini, since the system $|F|$ has no base point. If $F^{2}=2$ or 4 , by Bertini the general fibre $A$ can be singular only in base points. If all fibres $A$ are singular in some base point, then necessarily $A^{2} \geq 4$. In this case the base point is a double point on all fibres. Two distinct fibres cannot have a common tangent at this singularity. This implies that the general curve $A$ has an ordinary node at the base point and is smooth in all other points. The curve $\pi(A)$ is irreducible of degree $a$.

We apply the adjunction formula on $X$ :

$$
2 p_{a}(A)-2=A^{2}+2 a, \quad p_{a}(A)=a+\frac{1}{2} A^{2}+1 .
$$

If $A^{2}=0$ or 2 , the general curve $A$ is smooth with $p_{g}(A)=p_{a}(A)$. For $A^{2}=4$ the general curve $A$ may have an ordinary node, hence $p_{g}(A) \geq p_{a}(A)-1$. In these three cases we have the following lower bounds for $p_{g}(A)$ :

$$
\begin{array}{c|ccc}
A^{2} & 0 & 2 & 4 \\
\hline p_{g}(A) & a+1 & a+2 & a+2
\end{array}
$$

Since $a \geq 1$, necessarily $p_{g}(A) \geq 2$. All irreducible space curves of degrees $\leq 4$ are rational or elliptic, unless they are plane quartics, in which case we are done. This already shows $a \geq 5$. We apply the Castelnuovo-bound [ACGH, p. 116] for the irreducible curve $\pi(A)$, if it is non-planar:

$$
\begin{array}{c|ll}
a & 5 & 6 \\
\hline p_{g}(A) \leq & 2 & 4
\end{array}
$$

It cannot be met by our curve, and this curve must be planar.

Now we aim at the final contradiction. Consider some curve $B+F \in\left|\mathcal{C}^{\prime}\right|$ with $\pi(F)$ an irreducible planar quintic. The plane $S_{1}$ of $\pi(F)$ cannot touch $Y$ along $\pi(F)$. The curve $3 \cdot(B+F)$ has the class

$$
\mathcal{O}_{X}(3)-\sum_{\nu=1}^{n}\left(2 E_{\nu}^{\prime}+E_{\nu}^{\prime \prime}\right)
$$

So there will be a cubic surface $S \subset \mathbb{P}_{3}$ pulling back on $X$ to the curve $3(B+F)+\sum\left(2 E_{\nu}^{\prime}+E_{\nu}^{\prime \prime}\right)$. In particular $S$ will meet the plane $S_{1}$ of $\pi(F)$ in this quintic curve. Hence $S=S_{1}+S_{2}$ splits off $S_{1}$. The residual quadric $S_{2} \subset S$ will touch the surface $Y$ along the curve $\pi(F)$. So even $S_{2}$ splits off the plane $S_{1}$, say $S_{2}=S_{1}+S_{3}$ with another plane $S_{3}$. And again $S_{3}$ will contain the plane quintic, hence it coincides with $S_{1}$. We have shown $S=3 S_{1}$. Then $S_{1}$ must contain the whole curve $\pi(B+F)$. This means that $\pi(B+F)$ is a plane section of $Y$, contradicting lemma $1.1 \mathrm{c})$. This proves prop. 1.2 .

Theorem 1.1 Each non-empty 3-divisible set of cusps on a sextic surface $Y$ (with at most rational double points) contains at least 18 points. 
Proof. By Riemann-Roch

$$
2=h^{0}\left(\mathcal{C}^{\prime}\right)+h^{0}\left(\mathcal{C}^{\prime \prime}\right) \geq \chi\left(\mathcal{C}^{\prime}\right)=8-\frac{n}{3} . \square
$$

Theorem 1.2 Assume that the surface $Y \subset \mathbb{P}_{3}$ of degree 4,5 or 6 carries a 3-divisible set of cusps of the minimal number 6,12 or 18 . Then

- there are two distinct cubic surfaces $S^{\prime}$ and $S^{\prime \prime}$ passing through these cusps and touching $Y$ to the third order away from these cusps along two curves $\pi\left(C^{\prime}\right)$ and $\pi\left(C^{\prime \prime}\right)$;

- there is a quadric surface $S$ cutting out on $Y$ the two curves $\pi\left(C^{\prime}\right)$ and $\pi\left(C^{\prime \prime}\right)$.

Proof. By prop. 1.1 the classes $\mathcal{C}^{\prime}$ and $\mathcal{C}^{\prime \prime}$ are effective. Choose divisors $C^{\prime} \in\left|\mathcal{C}^{\prime}\right|, C^{\prime \prime} \in\left|\mathcal{C}^{\prime \prime}\right|$. Then

$$
3 C^{\prime} \sim \mathcal{O}_{X}(3)-\sum\left(2 E_{\nu}^{\prime}+E_{\nu}^{\prime \prime}\right), \quad 3 C^{\prime \prime} \sim \mathcal{O}_{X}(3)-\sum\left(E_{\nu}^{\prime}+2 E_{\nu}^{\prime \prime}\right) .
$$

This implies that there are cubic surfaces $S^{\prime}$ and $S^{\prime \prime}$ cutting out on $Y$ the divisors $3 \pi\left(C^{\prime}\right)$ and $3 \pi\left(C^{\prime \prime}\right)$ respectively. If these surfaces would coincide, then so would their divisor $\pi\left(C^{\prime}\right)=\pi\left(C^{\prime \prime}\right)$ of contact with $Y$. Let $C \subset X$ be the proper transform of this divisor. Then

$$
C^{\prime}=C+E^{\prime}, \quad C^{\prime \prime}=C+E^{\prime \prime}
$$

with integral linear combinations $E^{\prime}, E^{\prime \prime}$ of exceptional divisors. But then

$$
C^{\prime}-C^{\prime \prime} \sim \frac{1}{3} \sum\left(-2 E_{\nu}^{\prime}-E_{\nu}^{\prime \prime}+E_{\nu}^{\prime}+2 E_{\nu}^{\prime \prime}\right)=\frac{1}{3} \sum\left(E_{\nu}^{\prime \prime}-E_{\nu}^{\prime}\right) \sim \mathcal{L}^{\prime}-\sum E_{\nu}^{\prime}
$$

would be linearly equivalent to $E^{\prime}-E^{\prime \prime}$, a contradiction with lemma 1.1 a).

The divisor $C^{\prime}+C^{\prime \prime}$ on $X$ has the class

$$
\mathcal{O}_{X}(2)-\sum\left(E_{\nu}^{\prime}+E_{\nu}^{\prime \prime}\right)
$$

Hence $\pi\left(C^{\prime}+C^{\prime \prime}\right)$ is the intersection of $Y$ with a quadric surface $S$.

\section{Equations}

Consider the surface $Y$ of degree 4,5 or 6 with its contact cubics

$$
S^{\prime}: s^{\prime}=0, \quad S^{\prime \prime}: s^{\prime \prime}=0
$$

as constructed at the end of section 1 . The product $s^{\prime} \cdot s^{\prime \prime}$ vanishes on the divisor $\pi\left(C^{\prime}+C^{\prime \prime}\right)$ cut out on $Y$ by some quadric surface

$$
S: s=0 \text {. }
$$

In particular $s^{\prime} \cdot s^{\prime \prime} / s^{3}$ is constant on $Y$. This constant may be squeezed e.g. into $s^{3}$. Then the sextic polynomial

$$
s^{\prime} \cdot s^{\prime \prime}-s^{3}
$$

vanishes on $Y$. Unless it vanishes identically on $\mathbb{P}_{3}$, this gives a sextic equation $s^{\prime} \cdot s^{\prime \prime}-s^{3}=0$ which defines in degree 
4 the surface $Y$ together with a residual quadric,

5 the surface $Y$ together with a residual plane,

6 the surface $Y$ properly.

We now analyze the situation

$$
s^{\prime} \cdot s^{\prime \prime}-s^{3}=0 \quad \text { identically on } \mathbb{P}_{3} .
$$

If here $s$ would be irreducible, it would divide $s^{\prime}$ and $s^{\prime \prime}$, say

$$
s^{\prime}=s \cdot l^{\prime}, \quad s^{\prime \prime}=s \cdot l^{\prime \prime} \quad \text { with } \quad \operatorname{deg}\left(l^{\prime}\right)=\operatorname{deg}\left(l^{\prime \prime}\right)=1 .
$$

But then $l^{\prime} \cdot l^{\prime \prime}$ would divide $s$, a contradiction. So necessarily $s=l^{\prime} \cdot l^{\prime \prime}$ is reducible and $s^{\prime} \cdot s^{\prime \prime}=\left(l^{\prime} \cdot l^{\prime \prime}\right)^{3}$. Here $s^{\prime}=$ const $\cdot\left(l^{\prime}\right)^{3}$ or $s^{\prime \prime}=$ const $\cdot\left(l^{\prime \prime}\right)^{3}$ would contradict lemma $\left.1.1 \mathrm{~b}\right)$. W.l.o.g. we therefore may assume

$$
s^{\prime}=\left(l^{\prime}\right)^{2} \cdot l^{\prime \prime}, \quad s^{\prime \prime}=l^{\prime} \cdot\left(l^{\prime \prime}\right)^{2}, \quad l^{\prime}, l^{\prime \prime} \text { linearly independent. }
$$

Let $H^{\prime}$ and $H^{\prime \prime} \subset \mathbb{P}_{3}$ be the planes of equation $l^{\prime}=0, l^{\prime \prime}=0$ respectively. Let $I=H^{\prime} \cap H^{\prime \prime}$ be their line of intersection. Consider the divisors cut out on $Y$ by these two planes. If $Y$ contains the line $I$, let $i^{\prime}$, resp. $i^{\prime \prime}$ be the multiplicities of $I$ in these divisors. So

$$
H^{\prime} . Y=A^{\prime}+i^{\prime} I, \quad H^{\prime \prime} . Y=A^{\prime \prime}+i^{\prime \prime} I,
$$

with $I \not \subset A^{\prime}, A^{\prime \prime}$ and $i^{\prime}=i^{\prime \prime}=0$ if $I \not \subset Y$. Recall from section 1

$$
2 A^{\prime}+\left(2 i^{\prime}+i^{\prime \prime}\right) I+A^{\prime \prime}=S^{\prime} . Y=3 \pi\left(C^{\prime}\right), \quad A^{\prime}+\left(i^{\prime}+2 i^{\prime \prime}\right) I+2 A^{\prime \prime}=S^{\prime \prime} . Y=3 \pi\left(C^{\prime \prime}\right) .
$$

This implies $A^{\prime}=3 B^{\prime}$ and $A^{\prime \prime}=3 B^{\prime \prime}$. In particular both the divisors $\pi\left(C^{\prime}\right)$ and $\pi\left(C^{\prime \prime}\right)$ will be non-reduced.

Lemma 2.1 a) If $d=4$, the divisors $C^{\prime}$ and $C^{\prime \prime}$ can be chosen such that the sextic $s^{\prime} \cdot s^{\prime \prime}-s^{3}$ will not vanish identically.

b) If $d=5$ the situation that $s^{\prime} \cdot s^{\prime \prime}-s^{3}$ vanishes identically does not occur.

Proof. a) If $d=4$ we know

$$
\chi\left(\mathcal{C}^{\prime}\right)=\chi\left(\mathcal{C}^{\prime \prime}\right)=2 .
$$

For $C^{\prime} \in\left|\mathcal{C}^{\prime}\right|$ the image $\pi\left(C^{\prime}\right)$ has degree 4 . If $C^{\prime}$ is chosen generically, this image cannot consist of rational curves only. It is an elliptic quartic curve, or an elliptic cubic plane curve together with a line. In particular it is reduced. Hence $s^{\prime} \cdot s^{\prime \prime}-s^{3}$ will not vanish identically on $\mathbb{P}_{3}$.

b) If $d=5$, both the divisors

$$
Y \cdot H^{\prime}=3 B^{\prime}+i^{\prime} I, \quad Y \cdot H^{\prime \prime}=3 B^{\prime \prime}+i^{\prime \prime} I
$$

have degree 5. This implies $i^{\prime} \geq 2$ and $i^{\prime \prime} \geq 2$. But this means, the surface $Y$ touches both the planes $H^{\prime}$ and $H^{\prime \prime}$ along $I$. Then $I$ will be a double line on $Y$. Since $Y$ has isolated singularities only, this is impossible. 
Theorem 2.1 Quintics with 12 three-divisible cusps (and else at most rational double points) form an irreducible family.

Proof. Let $Y$ be a quintic with 12 three-divisible cusps. Choose polynomials $s^{\prime}, s^{\prime \prime}, s$ as in thm. 1.2 such that $s^{\prime} \cdot s^{\prime \prime}-s^{3}$ vanishes on $Y$ together with some residual plane $R$. By equivariance under the projective group it suffices to show that the equations $s^{\prime} \cdot s^{\prime \prime}-s^{3}$ vanishing on a fixed plane $R$ form an irreducible family. So fix the plane $R: x_{0}=0$. We have to consider separately different (mostly degenerate) cases:

Case 1: The quadric $S: s=0$ contains the plane $R$.

Subcase 1a) $R=S$ and, say, $s=x_{0}^{2}, s^{\prime}=x_{0} \cdot q^{\prime}$. Then $Y$ has an equation $f=q^{\prime} \cdot s^{\prime \prime}-x_{0}^{5}=0$ with all the twelve cusps on $R$. The set $q^{\prime}=s^{\prime \prime}=x_{0}=0$ consists of singularities of $Y$, hence this set is discrete. In particular it contains at most six points, which can be cusps of $Y$. So there must be at least six more cusps on $Y$ where $s^{\prime \prime}=0$ but $q^{\prime} \neq 0$. But there $d\left(q^{\prime} \cdot s^{\prime \prime}\right)=q^{\prime} \cdot d s^{\prime \prime}$ vanishes if and only if $d s^{\prime \prime}=0$, i.e., if the cubic surface $S^{\prime \prime}$ itself is singular. If the curve $s^{\prime \prime}=x_{0}=0$ is reduced, this can happen in at most three points. So the curve $s^{\prime \prime}=x_{0}=0$ must be non-reduced. It cannot consist of a threefold line, because on this line there could be at most four singularities of $Y$. And if it consists of a double line together with a simple line, on the simple line there cannot be a singularity of $S^{\prime \prime}$. Again there are at most four singularities of $Y$ on the double line, not enough. This excludes subcase 1a).

Subcase 1b) The quadric $S$ splits off $R$, say $s=x_{0} \cdot x_{1}$ and $f=q^{\prime} \cdot s^{\prime \prime}-x_{0}^{2} \cdot x_{1}^{3}$. Denote by $P$ the plane $x_{1}=0$ and by $L$ the line $P \cap R$. Again the set $q^{\prime}=s^{\prime \prime}=x_{1}=0$ is singular on $Y$, hence discrete, and it can contain at most six cusps of $Y$. At least six more cusps of $Y$ must lie on the curve $C: s^{\prime \prime}=x_{0}=0$ away from $q^{\prime} \mid L=0$. Along this curve $C$, away from $L$ and $q^{\prime}=0$ locally $x_{0}^{2}\left|Y \sim s^{\prime \prime}\right| Y$ is a third power. This implies $x_{0} \mid Y$ itself is a third power. Hence in those points $S^{\prime \prime}$ and $R$ touch. For the intersection curve $S^{\prime \prime} . R$ the possibilities are $(M \subset R$ a line $\neq L)$ :

- $S^{\prime \prime} \cdot R=3 M$ : On $M$ there are at most four singularities of $Y$ with no further singularities on $L$.

- $S^{\prime \prime}=2 M+L$ : Again on $M$ there are at most four singularities of $Y$. Away from $M$ on $L$ we have $d s^{\prime \prime} \neq 0$ and singularities could occur only in points where $q^{\prime}=0$. But those points belong to the $\leq 6$ points in $q^{\prime}=s^{\prime \prime}=x_{1}=0$ considered already.

- $S^{\prime \prime} \cdot R=3 L$ : Again there are at most four singularities of $Y$ on this line.

In all these cases there are less than 12 cusps.

Case 2: The quadric $S$ meets $R$ in two distinct lines, say $L_{1}: x_{0}=x_{1}=0$ and $L_{2}: x_{0}=$ $x_{2}=0$. So $s=x_{1} \cdot x_{2}+x_{0} \cdot l$.

Subcase 2a) $s^{\prime}\left|R=x_{1}^{3}\right| R$ and $s^{\prime \prime}\left|R=x_{2}^{3}\right| R$. This is the general case. It is analyzed in [BR, thm.1.1] There it is shown that for general choice of the equation the surface $Y$ indeed has twelve cusps away from $R$ and is smooth in all its other points. That surfaces with such an equation form an irreducible family, this is quite obvious. 
Subcase 2b): $s^{\prime}\left|R=x_{1}^{2} \cdot x_{2}\right| R$ and $s^{\prime \prime}\left|R=x_{1} \cdot x_{2}^{2}\right| R$. Then $s^{\prime}=x_{1}^{2} \cdot x_{2}+x_{0} \cdot q^{\prime}, s^{\prime \prime}=x_{1} \cdot x_{2}^{2}+x_{0} \cdot q^{\prime \prime}$ and $Y$ is defined by

$$
f:=x_{1}^{2} x_{2} q^{\prime \prime}+x_{1} x_{2}^{2} q^{\prime}+x_{0} q^{\prime} q^{\prime \prime}-3 x_{1}^{2} x_{2}^{2} l-3 x_{0} x_{1} x_{2} l^{2}-x_{0}^{2} l^{3} .
$$

This equation vanishes on both the lines $L_{i}$, hence both lines belong to $Y$. We claim $q^{\prime} \mid L_{1}=0$ (and similarly $q^{\prime \prime} \mid L_{2}=0$ ). Indeed, this is true if $S^{\prime}$ is singular along $L_{1}$. And if $S^{\prime}$ is smooth along $L_{1}$ it touches there $Y$ and $R$ simultaneously. This implies that $Y$ and $R$ touch along $L_{1}$. And by the form of $f$, also this proves the claim. So in fact $Y$ is singular along both lines, contradiction.

Case 3: The quadric $S$ meets $R$ in a repeated line, say $s=x_{1}^{2}+x_{0} \cdot l$ and and $s^{\prime}=$ $x_{1}^{3}+x_{0} \cdot q^{\prime}, s^{\prime \prime}=x_{1}^{3}+x_{0} \cdot q^{\prime \prime}$. Then $Y$ is defined by the polynomial

$$
f:=x_{1}^{3}\left(q^{\prime}+q^{\prime \prime}\right)+x_{0} q^{\prime} q^{\prime \prime}-3 x_{1}^{4} l-3 x_{0} x_{1}^{2} l^{2}-x_{0}^{2} l^{3} .
$$

By letting the lines $L_{1}$ and $L_{2}$ in subcase 2a) move together one proves that the surfaces in this family lie in the closure of the irreducible family from subcase 2a). However there is still some subtile point, namely, whether the twelve cusps from 2a) converge to the twelve 3-divisible cusps on the surfaces from case 3 . Therefore let us analyse the possible configuration of cusps in case 3:

By the form of $f$ the line $L: x_{0}=x_{1}=0$ lies on $Y$. Along this line $S^{\prime}$ and $S$, as well as $S^{\prime \prime}$ and $S$ touch. So off $R$ the (there necessarily discrete) set $S \cap S^{\prime} \cap S^{\prime \prime}$ contains at most eight points. And they are cusps on $Y$ if and only if this intersection is transversal, i.e., if it are eight points indeed. Additional cusps of $Y$ can lie only on $S^{\prime} \cap S^{\prime \prime} \cap R=L$. Since $\left(\partial_{0} f\right) \mid L=q^{\prime} \cdot q^{\prime \prime}$ there are indeed four more singularities of $Y$ on this line, for general choice of $q^{\prime}$ and $q^{\prime \prime}$. That these are cusps, e.g. the points of $L$ where $q^{\prime}=0$, this follows from the SQH-criterion [BW] with the weights

$$
w t\left(x_{1}\right)=\frac{1}{3}, \quad w t\left(x_{0}\right)=w t\left(q^{\prime}\right)=\frac{1}{2} .
$$

It remains to show for a family $Y_{t}$ of surfaces from subcase $2 \mathrm{a}$ ) converging to a surface $Y_{0}$ considered here, with twelve cusps, that the twelve cusps on $Y_{t}$ converge point by point to the twelve cusps on $Y_{0}$. If this would not be the case, at least two cusps $P_{1}(t)$ and $P_{2}(t)$ on $Y_{t}$ would converge to the same cusp $P_{0}$ on $Y_{0}$. Now fix a small sphere $S$ around $P_{0}$ containing $P_{1}(t)$ and $P_{2}(t)$ but no other singularities of $Y_{0}$. We consider the Milnor number $\mu\left(P_{0}\right)=2$ of $Y_{0}$ at $P_{0}$. By Milnor's definition [Mi, p. 59] it is the degree of the map of $S$ onto the unit sphere, defined by $g /\|g\|$ with $g=\left(\partial_{0} f_{0}, \partial_{1} f_{0}, \partial_{2} f_{0}\right)$, where $f_{0}=0$ is a local equation for $Y_{0}$ and the derivatives are taken w.r.t. some local coordinates. By continuity this is the same as the number defined by $f_{t}$, where $f_{t}=0$ for small $t$ is a local equation of $Y_{t}$, such that $f_{t}$ converges to $f_{0}$. But by [Mi, p.112] this is at least the sum of the Milnor numbers of all singularities of $Y_{t}$ contained in the fixed sphere. This sum is $\geq \mu\left(P_{1}(t)\right)+\mu\left(P_{2}(t)\right)=4$, contradiction.

Now we turn to degree 6 . If $s^{\prime} \cdot s^{\prime \prime}-s^{3}$ does not vanish identically on $\mathbb{P}_{3}$, then $s^{\prime} \cdot s^{\prime \prime}-s^{3}=0$ is an equation for the sextic surface $Y$. However the other case is possible too: 
Theorem 2.2 Assume that the polynomial $s^{\prime} \cdot s^{\prime \prime}-s^{3}$ vanishes identically on $\mathbb{P}_{3}$. Then there are homogeneous polynomials $l^{\prime}, l^{\prime \prime}, g, f$ of degrees

$$
\operatorname{deg}\left(l^{\prime}\right)=\operatorname{deg}\left(l^{\prime \prime}\right)=1, \quad \operatorname{deg}(g)=2, \quad \operatorname{deg}(f)=4,
$$

such that $Y$ is defined by the equation

$$
l^{\prime} \cdot l^{\prime \prime} \cdot f-g^{3}=0
$$

Proof. Put $s^{\prime}=\left(l^{\prime}\right)^{2} \cdot l^{\prime \prime}$ and $s^{\prime \prime}=l^{\prime} \cdot\left(l^{\prime \prime}\right)^{2}$ as above. We know already, that these polynomials define divisors

$$
3 B^{\prime}+i^{\prime} I \text { on } H^{\prime}, \quad 3 B^{\prime \prime}+i^{\prime \prime} I \text { on } H^{\prime \prime} .
$$

Here $i^{\prime}=i^{\prime \prime}=0 \bmod 3$. If $i^{\prime} \neq 0$, then the line $I$ is contained in $Y$ and $Y$ touches $H^{\prime}$ along this line. But then $i^{\prime \prime} \neq 0$ too, and $Y$ also touches $H^{\prime}$ along $I$. So $Y$ would contain $I$ as a multiple curve, which was excluded. In this way we see $i^{\prime}=i^{\prime \prime}=0$.

Next we show that both the curves $B^{\prime}$ and $B^{\prime \prime}$ are reduced conics. Assume e.g. that $B^{\prime}$ is a double line. All 18 cusps of $Y$ lie on the union $H^{\prime} \cup H^{\prime \prime}$ of both the planes, i.e. on $B^{\prime} \cup B^{\prime \prime}$. But since $Y$ is not singular along a curve, it can have at most five cusps on the line $B^{\prime}$ and at most ten cusps on the conic $B^{\prime \prime}$, not enough.

Now we claim that there is a quadratic polynomial $g$ restricting to $H^{\prime}$ as an equation for $B^{\prime}$ and to $H^{\prime \prime}$ as an equation for $B^{\prime \prime}$. First observe that both the conics $B^{\prime}$ and $B^{\prime \prime}$ meet $I$ in the same point set $Y \cap I$. This set may consist of two distinct points or of one point counted twice. Let $b^{\prime}$ and $b^{\prime \prime}$ be two quadratic polynomials restricting to the planes $H^{\prime}$ and $H^{\prime \prime}$ as equations for $B^{\prime}$ and $B^{\prime \prime}$ respectively. Then $b^{\prime} \mid I$ and $b^{\prime \prime} \mid I$ differ by a constant factor. We may adjust one of the quadric polynomials such that

$$
b^{\prime}\left|I=b^{\prime \prime}\right| I
$$

This implies

$$
b^{\prime}\left|H^{\prime \prime}=b^{\prime \prime}\right| H^{\prime \prime}+\left(l^{\prime} \cdot h\right) \mid H^{\prime \prime}, \quad \operatorname{deg}(h)=1 .
$$

Then the quadratic polynomial

$$
g:=b^{\prime}-l^{\prime} h
$$

restricts to $H^{\prime}$ as $b^{\prime} \mid H^{\prime}$ and to $H^{\prime \prime}$ as $b^{\prime \prime} \mid H^{\prime \prime}$. In particular the quadric $g=0$ cuts out on $H^{\prime}$ the conic $B^{\prime}$ and on $H^{\prime \prime}$ the conic $B^{\prime \prime}$.

Let now $\varphi=0$ be an equation for $Y$. Then both the polynomials $\varphi$ and $g^{3}$ define on $H \cup H^{\prime}$ the divisor $3\left(B^{\prime}+B^{\prime \prime}\right)$. On the line $I$ both polynomials differ by a constant factor. We may adjust $\varphi$ by this factor, to obtain $\varphi\left|I=g^{3}\right| I$. This then implies that $\varphi=g^{3}$ on $H^{\prime} \cup H^{\prime \prime}$. So there is a quartic polynomial $f$ with $\varphi-g^{3}=l^{\prime} \cdot l^{\prime \prime} \cdot f$, or

$$
\varphi=l^{\prime} \cdot l^{\prime \prime} \cdot f-(-g)^{3}
$$

as we claimed.

It is a different question, whether sextic surfaces with 18 three-divisible cusps and of equations

$$
s^{\prime} \cdot s^{\prime \prime}-s^{3}=0, \quad \text { resp. } \quad l^{\prime} \cdot l^{\prime \prime} \cdot f-g^{3}
$$


with

$$
\operatorname{deg}\left(l^{\prime}\right)=\operatorname{deg}\left(l^{\prime \prime}\right)=1, \quad \operatorname{deg}(s)=\operatorname{deg}(g)=2, \quad \operatorname{deg}\left(s^{\prime}\right)=\operatorname{deg}\left(s^{\prime \prime}\right)=3, \quad \operatorname{deg}(f)=4
$$

exist indeed. But choose all these polynomials generically. Then a Bertini-type argument [BR] shows that the surfaces with these equations are smooth but for the $3 \cdot 3 \cdot 2=18$ intersection points

$$
s^{\prime}=s^{\prime \prime}=s=0
$$

in the first case, and the $2+4 \cdot 2+4 \cdot 2=18$ points

$$
l^{\prime}=l^{\prime \prime}=g=0, \quad l^{\prime}=f=g=0, \quad l^{\prime \prime}=f=g=0
$$

in the second case. We refer to [BR] for the fact, that these 18 points indeed form a 3-divisible set.

It is clear that the polynomials $\varphi=s^{\prime} \cdot s^{\prime \prime}-s^{3} \in H^{0}\left(\mathcal{O}_{\mathbb{P}_{3}}(6)\right)$ with $s, s^{\prime \prime} \in H^{0}\left(\mathcal{O}_{\mathbb{P}_{3}}(3)\right)$ and $s \in H^{0}\left(\mathcal{O}_{\mathbb{P}_{3}}(2)\right.$ varying arbitrarily form an irreducible variety. It contains of course polynomials $\varphi$ defining degenerate surfaces, namely

- $\varphi \equiv 0$,

- defining surfaces with more than 18 singularities,

- defining surfaces where the 18 singularities defined degenerate or come together.

But these polynomials form a Zariski-closed subset. After removing them one has an irreducible variety of sextic polynomials defining surfaces $Y$ which are non-degenerate. This proves that all non-degenerate sextic surfaces of equation $s^{\prime} \cdot s^{\prime \prime}-s^{3}=0$ as above form an irreducible family. The same argument of course works for the second type of equation. We refer to [BR] for the fact that these two families are disjoint.

\section{References}

[ACGH] E. Arbarello, M. Cornalba, P. A. Griffiths, J. Harris: Geometry of algebraic curves. Volume I, Springer (1985)

[B 1] W. Barth: K3 Surfaces with nine cusps. Geom. Dedic. 72, 171-178 (1998)

[B 2] W. Barth: On the classification of K3 surfaces with nine cusps. In Complex Analysis and Algebraic Geometry (T. Peternell, F.-O. Schreyer ed.), de Gruyter, 41-59 (2000)

[BPV] W. Barth, C. Peters, A. Van de Ven: Compact Complex Surfaces. Springer (1984)

[BR] W. Barth, S. Rams: Cusps and codes, preprint (2003)

[Br] E. Brieskorn: Über die Auflösung gewisser Singularitäten von holomorphen Abbildungen. Math. Ann. 166, 76-102 (1966) 
[BW] J.W. Bruce, C.T.C. Wall: On the classification of cubic surfaces, J.London M.Soc. 19, 245-249 (1979)

[E] S. Endrass: Minimal even sets of nodes. J.r.u.a. Math. 503, 87-108 (1998)

[KZ] J. Keum, D.-Q. Zhang: Fundamental groups of open K3 surfaces, Enriques surfaces and Fano 3-folds, J. Pure Appl. Algebra 170, 67-91 (2002)

[Mi] J. Milnor: Singular Points of Complex Hypersurfaces, Ann. Math. Studies 61, Princeton Univ. Press (1968).

[M] Y. Miyaoka: The maximal number of quotient singularities on surfaces with given numerical invariants, Math. Ann. 268, 159-171 (1984)

[R] S. Rams: On quartics with three-divisible sets of cusps, Manuscripta Math. 111, 29-41 (2003)

[T] S.-L. Tan: Cusps on some algebraic surfaces and plane curves, preprint (1999)

[V] A. N. Varchenko: On semicontinuity of the spectrum and an upper estimate for the number of singular points of a projective hypersurface, Soviet Math. Dokl. 27, No. 3, 735-739 (1983)

Wolf P. Barth. Mathematisches Institut der Universität, Bismarckstr. 11/2, D - 91054 Erlangen, e-mail: barth@mi.uni-erlangen.de

Sławomir Rams. Institute of Mathematics, Jagiellonian University, ul. Reymonta 4, PL-30-059 Kraków, POLAND, e-mail: rams@im.uj.edu.pl 